\title{
A NOTE ON GAMMA FUNCTIONS
}

\author{
by G. N. WATSON
}

Various improvements in the formula

$$
\frac{2}{\pi}=\frac{2}{1} \cdot \frac{2}{3} \cdot \frac{4}{3} \cdot \frac{4}{5} \cdot \frac{6}{5} \ldots \frac{2 n}{2 n-1} \cdot \frac{2 n}{2 n+1} \ldots
$$

which was discovered by Wallis in 1669 , were studied by $\mathrm{D}$. K. Kazarinoff in No. 40 of these Notes (December 1956). He began by quoting from textbooks the formula

$$
\frac{1.3 .5 \ldots(2 n-1)}{2.4 .6 \ldots(2 n)}=\frac{1}{\sqrt{ }\{\pi(n+\theta)\}}, \quad 0<\theta<\frac{1}{2} ; n=1,2, \ldots
$$

(I do not remember having seen this formula stated explicitly; but it, like the original formula, is an immediate consequence of taking $z=\frac{1}{2} \pi$ in the canonical product for $\sin$ 2.) He then sharpened this result by proving that the inequality satisfied by $\theta$ could be replaced by $\frac{1}{4}<\theta<\frac{1}{2}$. He deduced this inequality as a special case of the corresponding inequality for Gamma functions, establishing the latter by applying some rather elaborate analysis to an integral formula due to Legendre.

I have noticed that the results which he obtained (and more) are almost immediate consequences of a special case of the Gaussian formula for the hypergeometric function with final element equal to unity, as I now proceed to show.

We write

$$
\frac{\Gamma\left(x+\frac{1}{2}\right)}{\Gamma(x+1)} \equiv f(x) \equiv \frac{1}{\sqrt{\{x+\theta(x)\}}},
$$

taking $x+\frac{1}{2}$ positive (or zero) throughout the following work.

Then, by the formula just mentioned, we have

$$
\begin{aligned}
\theta(x)=-x+x \frac{\Gamma(x) \Gamma(x+1)}{\Gamma^{2}\left(x+\frac{1}{2}\right)} & =-x+x F\left(-\frac{1}{2},-\frac{1}{2} ; x ; 1\right) \\
& =\sum_{m=1}^{\infty} \frac{\left(-\frac{1}{2}\right)_{m}\left(-\frac{1}{2}\right)_{m}}{m !(x+1)_{m-1}}
\end{aligned}
$$

with the usual notation

$$
(z)_{0}=1, \quad(z)_{m}=z(z+1) \ldots(z+m-1), \quad(m=1,2,3, \ldots) ;
$$

the condition $x+\frac{1}{2} \geqslant 0$ amply secures the convergence of the series.

Now each term of the series

$$
\sum_{m=2}^{\infty} \frac{\left(-\frac{1}{2}\right)_{m}\left(-\frac{1}{2}\right)_{m}}{m !(x+1)_{m-1}}
$$


is positive and decreases as $x$ is increased. It follows immediately that $\theta(x)$ is a monotonic decreasing function of $x$; we also have

while

$$
\theta\left(-\frac{1}{2}\right)=\frac{1}{2}
$$

$$
\theta(0)=\pi^{-1}=0 \cdot 318 \ldots, \quad \theta(1)=4 \pi^{-1}-1=0 \cdot 273 \ldots
$$

We now consider what happens when $x \rightarrow \infty$. For $x \geqslant 1$ we have

$$
\begin{aligned}
0<\theta(x)-\frac{1}{4} & =\frac{1}{x+1} \sum_{m=2}^{\infty} \frac{\left(-\frac{1}{2}\right)_{m}\left(-\frac{1}{2}\right)_{m}}{m !(x+2)_{m-2}} \\
& \leqslant \frac{1}{x+1} \sum_{m=0}^{\infty} \frac{\left(-\frac{1}{2}\right)_{m}\left(-\frac{1}{2}\right)_{m}}{m !(3)_{m-2}} \\
& \rightarrow 0 \text { as } x \rightarrow \infty
\end{aligned}
$$

This establishes the sharpened theorem, namely $\theta(x)>\frac{1}{4}$ in place of $\theta(x)>0$ for a continuous variable $x\left(x \geqslant-\frac{1}{2}\right)$, which was discovered and proved by Kazarinoff for $x$ positive.

I have not tried to ascertain whether the monotonic property of $\theta(x)$ (which is evident by my method) can be obtained by Kazarinoff's method.

The inequalities

$$
\frac{1}{4} \leqslant \theta(x) \leqslant \frac{1}{2}, \quad\left(x \geqslant-\frac{1}{2}\right) ; \quad \frac{1}{4}<\theta(x) \leqslant \pi^{-1}, \quad(x \geqslant 0)
$$

are evident from the foregoing results.

We leave to the reader the task of deducing various inequalities from the relation

$$
x+\theta(x)=\frac{x^{2}}{x-\frac{1}{2}+\theta\left(x-\frac{1}{2}\right)}
$$

for appropriate ranges of values of $x$.

Next consider positive integral values $(n)$ of $x$ only, zero included.

For $n=0$ we have $\theta(0)=\pi^{-1}$, as already stated.

For $n=1,2,3, \ldots$ we have

$$
\begin{gathered}
\frac{1.3 .5 \ldots(2 n-1)}{2.4 .6 \ldots(2 n)} \sqrt{ } \pi=\frac{1}{\sqrt{\{n+\theta(n)\}}}, \\
\theta(1)=0 \cdot 273 \ldots \geqslant \theta(n)>1
\end{gathered}
$$

so that the change in the value of $\theta(n)$ as $n$ runs through these positive integral values is not particularly large.

I might mention that this is not my first encounter with the function here denoted by $f(x)$. It is proved by E. W. Hobson, Spherical and Ellipsoidal Harmonics (1931), \$192 that the function satisfies the rather weak inequality

$$
f(x)<1 / \sqrt{ }\left(x-\frac{1}{2}\right), \quad\left(x>\frac{1}{2}\right) .
$$

When I saw the paged proofs of the book, I remarked to Hobson that the inequality could be easily obtained from the modified form of the First Eulerian Integral

$$
f(x)=\frac{2}{\sqrt{ } \pi} \int_{0}^{\frac{1}{2} \pi} \cos ^{2 x} \theta d \theta=\frac{2}{\sqrt{ } \pi} \int_{0}^{\infty} \exp \left(-x t^{2}\right) \frac{t \exp \left(-\frac{1}{2} t^{2}\right) d t}{\sqrt{ }\left\{1-\exp \left(-t^{2}\right)\right\}}
$$


and he agreed with me; but it did not seem worth while going to the trouble and expense of replacing his work by mine.

By using the fairly obvious inequalities

$$
\sqrt{ }\left\{1-\exp \left(-t^{2}\right)\right\} \leqslant t, \frac{t \exp \left(-\frac{1}{4} t^{2}\right)}{\sqrt{ }\left\{1-\exp \left(-t^{2}\right)\right\}}=\frac{t}{\sqrt{ }\left(2 \sinh \frac{1}{2} t^{2}\right)} \leqslant 1,
$$

we have, for $x>-\frac{1}{4}$,

$$
\frac{2}{\sqrt{ } \pi} \int_{0}^{\infty} \exp \left\{-\left(x+\frac{1}{2}\right) t^{2}\right\} d t<f(x)<\frac{2}{\sqrt{ } \pi} \int_{0}^{\infty} \exp \left\{-\left(x+\frac{1}{4}\right) t^{2}\right\} d t,
$$

that is to say

$$
1 / \sqrt{ }\left(x+\frac{1}{2}\right)<f(x)<1 / \sqrt{ }\left(x+\frac{1}{4}\right) .
$$

These are the results given by Kazarinoff ; and Hobson's inequality is a weakened version of the right-hand inequality.

46 W ARWICK NEW Road

LEAMINGTON, WARWIOKSHIRE 